\title{
Parameterized Analysis of Zero Voltage Switching in Resonant Converters for Optimal Electrode Layout of Piezoelectric Transformers
}

\author{
Meyer, Kaspar Sinding; Andersen, Michael Andreas E.; Jensen, Flemming
}

Published in:

39th IEEE Annual Power Electronics Specialists Conference

Link to article, DOI:

10.1109/PESC.2008.4592323

Publication date:

2008

Document Version

Publisher's PDF, also known as Version of record

Link back to DTU Orbit

Citation (APA):

Meyer, K. S., Andersen, M. A. E., \& Jensen, F. (2008). Parameterized Analysis of Zero Voltage Switching in Resonant Converters for Optimal Electrode Layout of Piezoelectric Transformers. In 39th IEEE Annual Power Electronics Specialists Conference (pp. 2543-2548). IEEE. https://doi.org/10.1109/PESC.2008.4592323

\section{General rights}

Copyright and moral rights for the publications made accessible in the public portal are retained by the authors and/or other copyright owners and it is a condition of accessing publications that users recognise and abide by the legal requirements associated with these rights.

- Users may download and print one copy of any publication from the public portal for the purpose of private study or research.

- You may not further distribute the material or use it for any profit-making activity or commercial gain

- You may freely distribute the URL identifying the publication in the public portal 


\section{Parameterized Analysis of Zero Voltage Switching in Resonant Converters for Optimal Electrode Layout of Piezoelectric Transformers}

\author{
Kaspar Sinding Meyer ${ }^{1,2}$ and Michael A. E. Andersen ${ }^{1}$ \\ ${ }^{1}$ Technical University of Denmark \\ DTU Elektro \\ Elektrovej Building 325 \\ DK - 2800 Kgs. Lyngby, Denmark \\ Email: ksm@noliac.com and ma@elektro.dtu.dk
}

\author{
Flemming Jensen ${ }^{2}$ \\ ${ }^{2}$ Noliac A/S \\ Hejreskovvej 18 \\ DK - 3490 Kvistgaard, Denmark \\ Email: fj@noliac.com \\ Homepage: www.noliac.com
}

\begin{abstract}
Ring shaped PTs (Piezoelectric Transformers) are an attractive alternative to magnetics in power converters. The achievable energy efficiency is $98 \%$ and the power density is up to $30 \mathrm{~W} / \mathrm{cm}^{3}$. Additionally power supplies based on PTs display low levels of conducted and radiated EMI due to power conversion based on the piezoelectric effect. Rooted in the physics of this effect, both the in- and output terminal of a PT has a noticeable parasitic capacitance. In a common half-bridge power stage without any supporting magnetic components, the input parasitic capacitance can lead to hard switching losses that are in the range of the actual power rating of a specific PT. In this paper it is demonstrated how the electrode layout of a PT can be designed to enable ZVS (Zero Voltage Switching). This optimization is made simple with a novel set of accurate and simple symbolic equations which relates ZVS constraints to the physical electrode layout of a PT. These properties takes basis in the formulation of an equivalent electrical PT circuit that is valid around the frequency of operation.
\end{abstract}

\section{INTRODUCTION}

The characteristics of PTs are very different from magnetic transformers used in PWM converters, both in relation the physical principles on which they operate and how they should be implemented in a power supply. Figure 1 shows the measured transfer function of the 2005-09-05-A prototype made by Noliac A/S. The measurement displays a number of resonance peaks corresponding to frequencies at which a standing wave is physically present in the transformer.

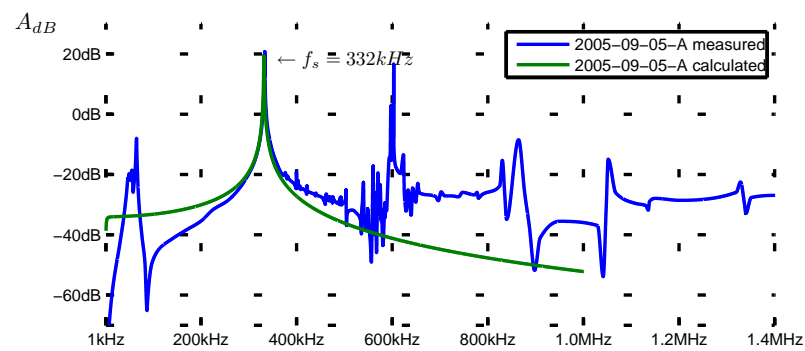

Fig. 1. Transfer function of 2005-09-05-A measured with a HP4194A $\stackrel{f}{\rightarrow} \rightarrow$ mpedande/gain-phase analyzer compared to an equivalent model of the fundamental resonance mode. The load is $5 k \Omega$.
Earlier references [1], [2], [3] has proven a proportional relation between the resonance frequency of operation and the power density of a PT. In Fig. 1 the 2005-09-05-A PT has two distinct resonance peaks respectively at $332 \mathrm{kHz}$ and $602 \mathrm{kHz}$, where the latter potentially has the highest power density. In terms of efficiency however, the spurious modes around $602 \mathrm{kHz}$ has a degrading effect and the mode at $332 \mathrm{kHz}$ is the optimal operating frequency.

The characteristics of piezoelectric transformers resembles a distributed network, but for a mathematical representation it is advantageous to model only the resonance mode of interest [4]. The equivalent circuit in Fig. 2 is used throughout the literature [4], [5], [3] and is useful for deriving properties of a PT, which is the objective of this paper. The equivalent transfer function for 2005-09-05-A is shown in Fig. 1 compared to the one measured.

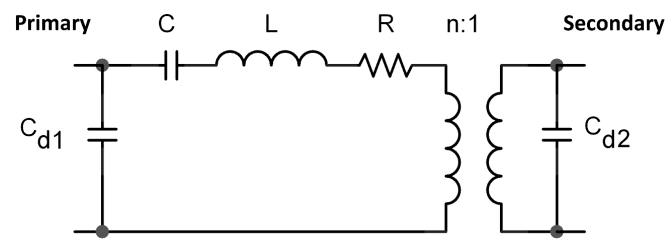

Fig. 2. Equivalent electrical circuit which is valid around one resonance mode.

The parameters can be extracted from the relation of the equivalent circuit to a set of primary and secondary side impedance measurements. By shorting the opposite terminal of the one being measured the equivalent circuit is simplified. The transfer function for the impedance seen from the primary side is derived in (1). It has one integrator, a complex pole pair responsible for the peak and a zero pair responsible for the notch in the impedance plot. To illustrate this a complete set of measurements for 2005-09-05-A is shown in Fig. 3. The values $C_{T 1}$ and $C_{T 2}$ shown in the figure are the two terminal 
capacitances given by (2).

$$
\begin{gathered}
Z_{P}(s)=\frac{L C s^{2}+C R s+1}{\frac{C C_{d 1}}{C+C_{d 1}} L s^{2}+\frac{C C_{d 1}}{C+C_{d 1}} R s+1} \cdot \frac{1}{\left(C+C_{d 1}\right) s} \\
C_{T 1}=C_{d 1}+\frac{C C_{d 2}}{n^{2} C+C_{d 2}}, \quad C_{T 2}=C_{d 2}+n^{2} \frac{C C_{d 1}}{C+C_{d 1}}
\end{gathered}
$$

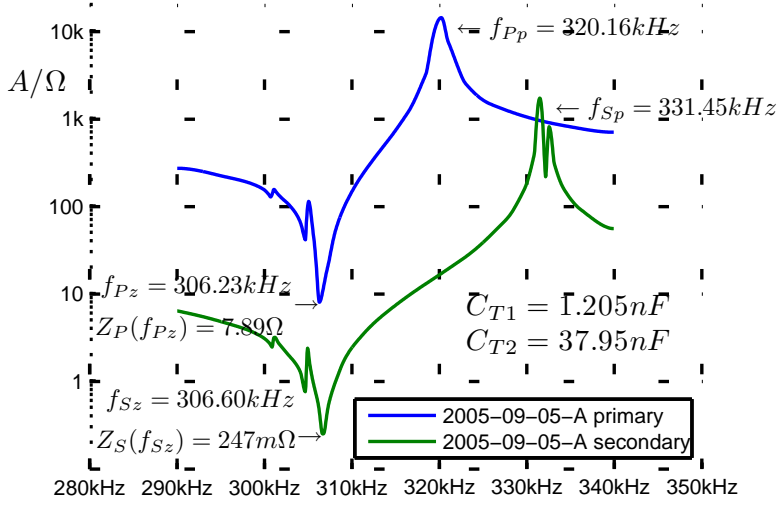

a)

$280 \mathrm{kHz}^{2} 290 \mathrm{kHz} 300 \mathrm{kHz} 310 \mathrm{kHz} 320 \mathrm{kHz}^{-} 330 \mathrm{kHz}^{-} 340 \mathrm{kHz}{ }^{-} 350 \mathrm{kHz}$

Fig. 3. Impedance seen from the primary and secondary terminals while the other side is shorted for Noliac 2005-09-05-A. The index p indicates seen from primary side, $\mathrm{s}$ seen from secondary side, ${ }_{\mathrm{z}}$ zero/notch and $\mathrm{p}$ pole/peak.

By comparing the impedance function with the general expression for a second order transfer function (3), the relation between the component parameters and the notch and the peak in the impedance measurement from Fig. 3 can be found.

$$
H_{2 n d}(s)=\frac{\frac{1}{\omega_{z}^{2}} s^{2}+\frac{1}{Q_{z} \omega_{z}} s+1}{\frac{1}{\omega_{p}^{2}} s^{2}+\frac{1}{Q_{p} \omega_{p}} s+1}
$$

Without any significant damping in the test setup, the quality factor of the transfer function is high and the relation from Fig. 3 to equivalent parameters become (4) to (8):

$$
\begin{gathered}
C_{d 1}=C_{T 1} \frac{\omega_{P z}^{2}}{\omega_{P p}^{2}}, \quad C_{d 2}=C_{T 2} \frac{\omega_{S z}^{2}}{\omega_{S p}^{2}} \\
C=C_{T 1}-C_{d 1} \\
L=\frac{1}{\omega_{P z}^{2} C} \\
n=\sqrt{L \omega_{S z}^{2}\left(C_{T 2}-C_{d 2}\right)} \\
R=Z_{P}\left(f_{P z}\right)
\end{gathered}
$$

The extracted equivalent parameters for Noliac-2005-09-05-A is shown in Fig. 4.

\section{Principle of Soft Switching}

The inductor-less half-bridge power stage illustrated in Fig. 4, has a low component count and is commonly used to drive PTs. While $C_{d 1}$ in the PT equivalent diagram is not needed for the operation of the circuit, it is an inherent part of the structure of a PT and needs to be incorporated into the operation of the circuit in order to avoid excessive switching losses. The drainsource capacitance of the two Mosfets has the same influence, but they are typically orders of a magnitude smaller than $C_{d 1}$ and can be disregarded.

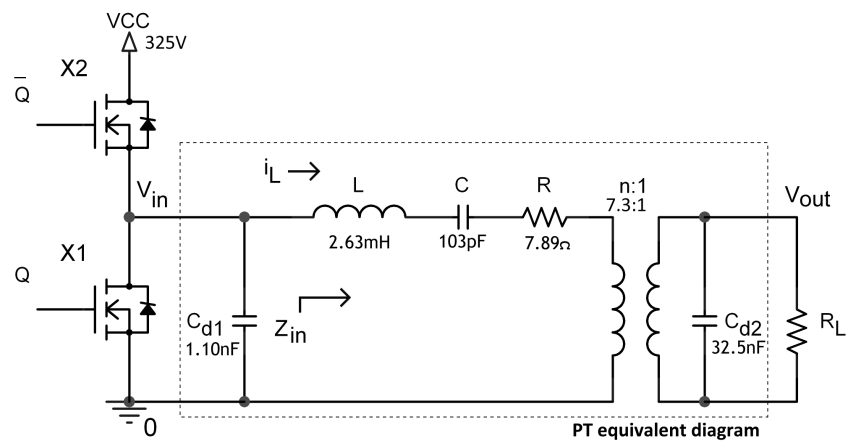

Fig. 4. Inductor-less half-bridge power stage connected to a PT equivalent diagram with values from the prototype Noliac-2005-09-05-A.

In hard switching mode the power switches $X_{1}$ and $X_{2}$ in Fig. 4 are driven at a $50 \%$ duty-cycle and produce a squarewave voltage at the input node $V_{i n}$ with an amplitude of $V_{C C}$. The losses associated with hard switching arise from the repetitive charging and discharging of the input capacitance $C_{d 1}$ and is given by equation (9). With the PT values for the ring-shaped prototype PT 2005-09-05-A by Noliac shown in Fig. 4, the switching losses alone become $42 \mathrm{~W}$ when driven from a rectified European mains voltage of $325 V_{D C}$.

$$
P_{\text {MOSFETs }}=f_{r} C_{d 1} V_{C C}^{2} \text { where } f_{r} \approx \frac{1}{2 \pi \sqrt{L C}}
$$

Given that the hard-switching losses are comparable to the maximum achievable power throughput of a PT, which can be calculated by (10) (assuming a matched load (23) and a ZVS waveform (12)), it is critical to apply means of soft-switching.

$$
P_{\text {max }}=V_{\text {in_rms }}^{2} \frac{2 C_{d 2}}{n^{2} \omega_{r}} \approx\left(V_{C C} \cdot 0.57\right)^{2} \frac{2 C_{d 2}}{n^{2} \sqrt{L C}}
$$

A. Principles of inductor-less soft switching with a half-bridge PT power stage

They key to avoid hard switching losses lies in the interpretation of the PT as a sinusoidal current source which is illustrated in Fig. 5.

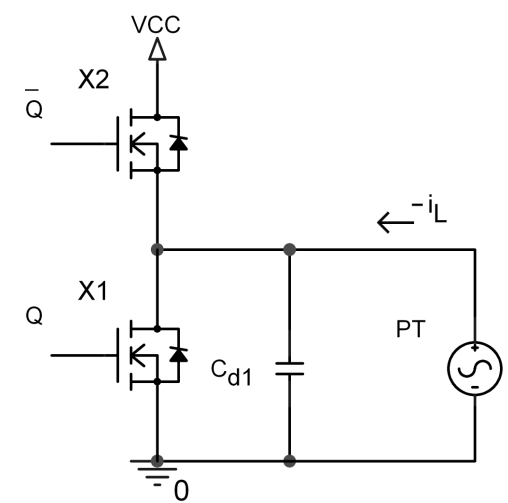

Fig. 5. The equivalent PT LCRC $\mathrm{C}_{\mathrm{d} 2}$ branch can be interrelated as a current source.

Given the fulfillment of the conditions in proposition 1, the equivalent PT current $-i_{L}$ will be able to fully charge and discharge $C_{d 1}$ when it would otherwise had been required by means of hard switching. 


\section{Proposition 1 Conditions for soft switching.}

1) The inverse PT current $-i_{L}$ has to be in advance of the input voltage $V_{i n}$.

2) $-i_{L}$ needs a sufficient amplitude to fully charge and discharge $C_{d 1}$.

3) The power switches are operated with an optimal dead time period determined by $\angle-i_{L}$.

The characteristic waveforms for a half-bridge power stage, with utilization of the PT as a current source for means of switching, is demonstrated in Fig. 6. Within the period where both transistors are turned OFF and when the Mosfet body diodes are not clamping the input voltage $V_{i n}$, the current through $C_{d 1}$ is $-i_{L}$. Given the fact that the switches are only turned $\mathrm{ON}$ when the voltage across them are zero, this way of soft switching is inherently called zero voltage switching.

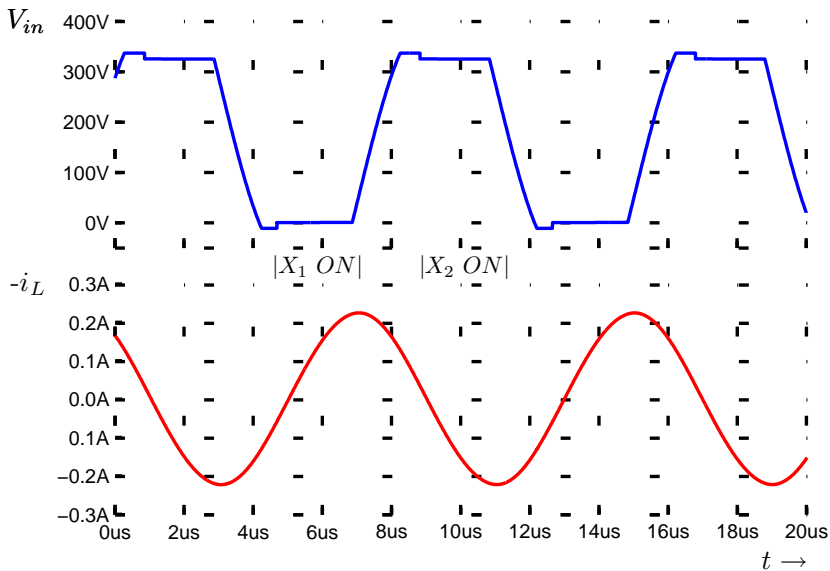

Fig. 6. Characteristic ZVS waveform with an exaggerated body diode forward voltage drop.

The items in proposition 1 can be accomplished when the circuit is operated at a frequency that is higher than the resonance frequency of the PT and with a PT internal electrode layout that is optimized for ZVS. In the following sections a mathematical expression for ZVS will be derived.

\section{MAThematicAl CONDitions FOR ZVS}

Two similar approaches to a mathematical model are found in [6] and [7]. The models assume that the input voltage is a square wave and that the inductor current is constant within the dead time period. Both assumptions are not in accordance with Fig. 6 and both mathematical models will result in too optimistic results. A more complete model is derived in [8], but common for all three references [6], [7] and [8] is that the derived expressions are large and do not provide a transparent relation between the parameters of a PT and its ZVS capabilities.

An improved and yet simple model can be formulated:

The LCR branch current $i_{L}$ from Fig. 4 has to be derived. Written in a phaser notation, the current can be expressed as the real part of the product between a complex sinusoidal and the PT input admittance $Z^{-1}{ }_{i n}(11)$ shown in Fig. 4.
This has cosinus as natural base and the phase shift is given by $\angle Z_{i n}^{-1}$. The term $A_{f}$ relates the supply voltage $V_{C C}$ to the fundamental fourier component of the input voltage $V_{\text {in }}$ assuming a worst case dead time of $1 /(4 f)$.

$$
\begin{array}{r}
i_{L}(t)=V_{C C} A_{f} \boldsymbol{R e}\left(e^{j \omega t} \frac{1}{Z_{i n}}\right) \\
\text { where } A_{f}=\frac{4 \sqrt{2}}{\pi^{2}} \approx 0.57
\end{array}
$$

The measure for the ZVS capability of a PT, is the peak resonant charge voltage $V_{p}(\omega)$ across the input capacitance $C_{d 1}$ at a given frequency with an optimal dead-time period, assuming that the reverse recovery diodes are not present. If this voltage exceeds the supply voltage $V_{C C}$, ZVS can be achieved. The solution is provided by the integral in equation (13).

$$
V_{P}(\omega)=\int_{t_{1}}^{t_{2}}-i_{L}(t) \frac{A_{f}}{C_{d} 1} d t
$$

With cosinus as the natural base, the first switching flank is symmetrical around $t=\frac{\pi}{2 \omega}$ (see Fig. 6) and the boundaries in which ZVS is possible are (14).

$$
t_{1}=\frac{\pi}{2 \omega}-\frac{\angle Z_{i n}}{\omega} \quad t_{2}=\frac{\pi}{2 \omega}+\frac{\angle Z_{i n}}{\omega}
$$

In phaser notation the integral in (13) reduces to a division with $j \omega$. This makes it possible to write the resonant peak voltage $V_{p}$ as in (15). Using the identity that the complex exponential $e^{j \pi / 2}$ represents a $90^{\circ}$ phase shift, i.e. multiplication with $j$, equation (15) reduces to (16). In addition to this, the phase shift $e^{j \angle Z_{i n}}$ in (16) is the equivalent of multiplication with the complex number $Z_{i n}$ factorized to a unity length (17).

$$
\begin{gathered}
V_{P}(\omega)= \\
\frac{V_{C C} A_{f}}{C_{d 1}} \boldsymbol{R} \boldsymbol{e}\left[\frac{1}{j \omega Z_{i n}}\left(e^{j \omega\left(\frac{\pi}{2 \omega}+\frac{\angle Z_{i n}}{\omega}\right)}-e^{j \omega\left(\frac{\pi}{2 \omega}-\frac{\angle Z_{i n}}{\omega}\right)}\right)\right] \\
=\frac{V_{C C} A_{f}}{C_{d 1}} \boldsymbol{R e}\left[\frac{1}{j \omega Z_{i n}}\left(j e^{j \angle Z_{i n}}-j e^{-j \angle Z_{i n}}\right)\right] \\
=\frac{V_{C C} A_{f}}{C_{d 1}} \boldsymbol{R} \boldsymbol{e}\left[\frac{1}{\omega Z_{i n}}\left(\frac{Z_{i n}}{\left|Z_{i n}\right|}-\frac{Z_{i n}^{*}}{\left|Z_{i n}\right|}\right)\right]
\end{gathered}
$$

In order to evaluate the real part of the complex equation for $V_{P}$, the input impedance $Z_{\text {in }}$ has to be divided into a real $\alpha$ and an imaginary part $\beta$. From expressions (15) to (17) it is recognized that the peak voltage is a linear with respect to $V_{C C}$, thus the ZVS condition $V_{P} \geq V_{C C}$ can be substituted with $\dot{V}_{P} \geq 100 \%$ where $\dot{V}_{P}=V_{P} / V_{C C}$. This relates into the final equation (21) for testing the ZVS properties of a PT in relation to frequency. Additionally the optimal dead time can 
be derived (22).

$$
\begin{aligned}
Z_{i n} & =\alpha+j \beta \\
\alpha & =R+\frac{R_{L} n^{2}}{\left(R_{L} C_{d 2} \omega\right)^{2}+1} \\
\beta & =\omega-\frac{1}{\omega C}-\frac{w C_{d 2}\left(R_{L} n\right)^{2}}{\left(\omega R_{L} C d 2\right)^{2}+1} \\
\dot{V}_{P}(\omega) & =\frac{4 \sqrt{2}}{\pi^{2} C_{d 1}} \cdot \frac{2 \beta^{2}}{\omega\left(\alpha^{2}+\beta^{2}\right)^{3 / 2}} \\
t_{\text {dead_time }} & =\frac{2}{\omega} \tan ^{-1}\left(\frac{\beta}{\alpha}\right)
\end{aligned}
$$

For a given set of PT parameters the derived resonant peak voltage (21) is an expression which is a function of frequency together with the load resistance and Fig. 7 serves to illustrate the maximum value as a function of load resistance for the PT 2005-09-05-A. From the plot a distinct minimum can be identified at the point of what is known as the matched load resistance $\dot{R}_{L}$. This is a resistive load which equals the output impedance of a PT (23) and has the property that it maximizes the efficiency of a PT [9], [8], [4]. In most cases the excitation frequency $\omega$ can be approximated with the resonance frequency $\omega_{r}$ (24) given the fact that the transformer is always operated in the close proximity of this frequency.

$$
\begin{gathered}
\dot{R}_{L}=\frac{1}{\omega C_{d 2}} \approx \frac{1}{\omega_{r} C_{d 2}} \\
\omega_{r}=\sqrt{\frac{2 C_{d 2}+n^{2} C}{2 L C_{d 2} C}} \approx \frac{1}{\sqrt{L C}}
\end{gathered}
$$

In relation to ZVS the matched load resistance provides the maximum achievable damping of the transformer, in which it serves as an important worst case scenario to work with. In this case the real and imaginary part of the PT input impedance (19), (20) reduces to (25) and the peak resonant charge voltage becomes solely dependent of the excitation frequency.

$$
\alpha=R+\frac{N^{2}}{2 \omega C_{d 2}}, \quad \beta=2 L\left(\omega-\omega_{r}\right)
$$

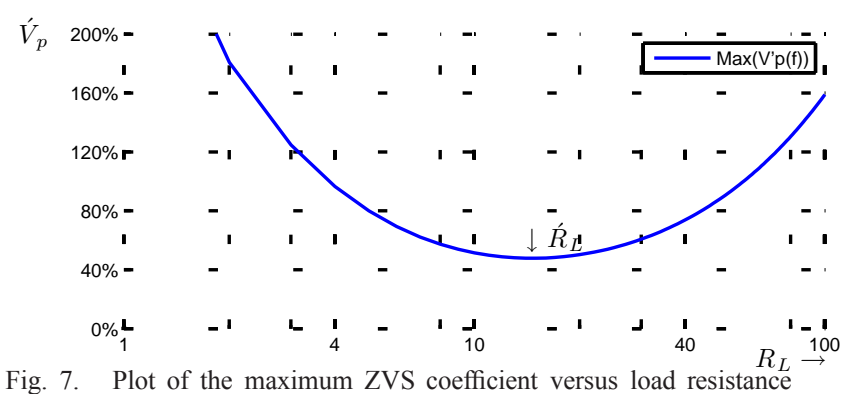
using the equivalent parameters for Noliac 2005-09-05-A PT.

In Fig. 8 the frequency dependent peak resonant charge voltage $V_{p}$ is plottet for two different transformers. In terms of optimization, the maximum point of ${ }^{\prime} V_{p}$ serves as a measure of ZVS capabilities of a PT. This value can be found using the chain rule for differentiation (26) and the peak resonant charge voltage relates into (28). As the equivalent loss resistance $R$ from the equivalent diagram provides no significant damping, the top point can be approximated by (29) which will be denoted the ZVS Coefficient $\hat{V} p$.

$$
\begin{gathered}
0=\frac{\delta \hat{V}_{P}}{\delta \omega}=\frac{\delta \hat{V}_{P}^{\prime}}{\delta \beta} \cdot \frac{\delta \beta}{\delta \omega} \\
\text { given } \frac{\delta \beta}{\delta \omega} \neq 0 \text { then } \frac{\delta V_{P}}{\delta \beta}=0 \Rightarrow \beta=\alpha \sqrt{2} \\
\dot{V}_{P}=\frac{1}{N^{2}} \cdot \frac{C_{d 2}}{C_{d 1}} \cdot \frac{32 \sqrt{6}}{9 \pi^{2}} \underbrace{\left(1-R \frac{\omega 2 C_{d 2}}{N^{2}}\right)}_{\approx 1} \\
\approx \frac{1}{N^{2}} \cdot \frac{C_{d 2}}{C_{d 1}} \cdot \frac{32 \sqrt{6}}{9 \pi^{2}}
\end{gathered}
$$

The derived worst case ZVS Coefficient $V_{p}$ (29) in a very simple equation and reveals that ZVS capability is only dependent on coupling ratio $n$ and the two parasitic capacitances $C_{d 1}$ and $C_{d 2}$. The ZVS capability is thereby independent of the resonance frequency $\omega_{r} \approx(\sqrt{L C})^{-1}$ together with the equivalent loss resistance $R$. Additionally it can be proven that for $\omega_{r}$ being constant, the ratio $L / C$ determines the ZVS bandwidth, but for simplicity this will not be treated in this paper.

The primary advantage of the simplified and novel ZVS coefficient (29) is to provide a worst case scenario for analyzing PTs and the simplicity of the symbolic expression can be used in the aid of designing a PT capable of ZVS. In the next section it will be shown, that the dimensions of the PT electrodes can directly be derived from the desired ZVS capabilities for a PT.

The plots in Fig. 8 display the relative ZVS resonant peak charge voltage versus frequency $\hat{V}_{P}(\omega)$ for respectively PT 2005-06-05-A with parameters from Fig. 4 and PT T1-2 with parameters shown in the caption of Fig. 8. The T1-2 is a disc shaped PT made by Face and 2005-06-05-A is a ring shaped PT invented by Noliac [1], which has been improved for efficiency and prevention of spiritus modes.

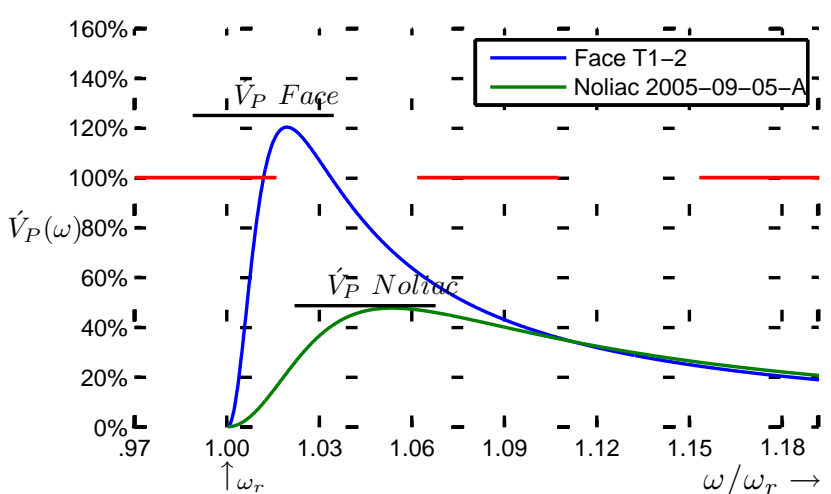

Fig. 8. Plot of the ZVS coefficient versus relative frequency for the two PTs Face T1-2 and Noliac 2005-06-05-A. Face T1-2 parameters: $\mathrm{R}=11.6 \Omega, \mathrm{Cd} 1=$ $1.55 \mathrm{nF}, \mathrm{Cd} 2=2.19 \mathrm{nF}, \mathrm{C}=120 \mathrm{pF}, \mathrm{L}=15.1 \mathrm{mH}, \mathrm{N}=1$

For both transformers the approximated ZVS Coefficient $\hat{V}_{P}$

(29) has been used to evaluate the maximum point (marked with a dashed line) and it can be verified that it coincides well with the maximum point of the original and frequency 
dependent expression $V_{p}(\omega)$ (21). The Face transformer has been designed for native ZVS as it can be verified from the plot.

From the PT equivalent diagram in Fig. 2 the efficiency with a matched load (23) can be derived (31). The Noliac transformer has been optimized solely for high efficiency and reaches $98 \%$ whereas the Face transformer peaks at $96 \%$. Although the two transformers have a different physical design and can not directly be compared, it is still apparent that ZVS does not come without a performance penalty.

$$
\begin{aligned}
\eta_{\dot{R}_{L}} & =\frac{1}{1+\omega \frac{2 R C_{d 2}}{n^{2}}} \\
& \approx 1-\frac{2 R C_{d 2}}{n^{2} \sqrt{L C}}
\end{aligned}
$$

By adding a series inductor to the Noliac PT design, the real and the imaginary parts of the input impedance changes in the equation for the resonant peak voltage (21) and this in turn enables ZVS. However doing so can be considered a temporary solution and in the next chapter a at set of guidelines PT ZVS optimization will be established.

\section{ZVS OPTIMIZATION OF THE PT ElECTRODE LAYOUT}

The ZVS coefficient is determined by the ratio of $n, C_{d 1}$ and $C_{d 2}$ as derived in (29). The parametric simplicity of this worst case equation, allows for a usefull a usefull approximative relation between ZVS properties and the physical layout to be drawn. In any given application, the transformer conversion ratio $n$ is constant, because it relates the input voltage to the output voltage. Starting from the ring-shaped PT which operates in its thickness mode, the conversion ration can under ideal conditions be found by (32), where $t_{p}$ and $t_{s}$ is the thickness of the primary and secondary electrode respectively. These dimensions are illustrated in the conceptual ring-shaped PT cross section view in Fig. 9a.

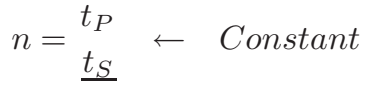

A ZVS enabled design is achieved by maximizing the ratio $C_{d 2} / C_{d 1}$ with the constraint that $n$ is fixed. If the presence

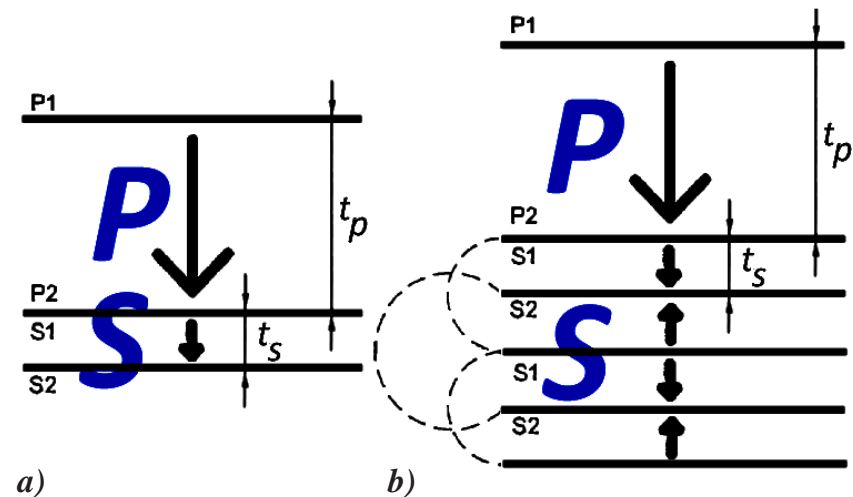

Fig. 9. Conceptual cross section view of a ring shaped PT. The arrows indicate the direction of polarization. a) Initial design. b) ZVS optimized design. of fringing fields is ignored, each terminal capacitance can be expressed by the well known plate capacitor equation used in (33). The relation between these capacitances and the equivalent parameters are given in (2) and it is apparent that if $C_{d 1} \gg C$ and $C_{d 2} \gg n^{2} C$ then (34) is a valid assumption to make.

$$
\begin{array}{r}
C_{T 1}=A_{P} \frac{\epsilon}{t_{P}}, \quad C_{T 2}=A_{S} \frac{\epsilon}{t_{S}} \\
C_{T 1} \approx C_{d 1}, \quad C_{T 2} \approx C_{d 2}
\end{array}
$$

This leads to the fact that ZVS is achieved by maximizing the area of the secondary electrode relative to the primary electrode in order to satisfy (35).

$$
\frac{A_{P}}{A_{S}}<\frac{32 \sqrt{6}}{n 9 \pi^{2}}
$$

This can for example be achieved in a design with large number of parallel secondary electrodes as illustrated in Fig. $9 \mathrm{~b}$. Usually the absolute size of the output capacitance is constrained to some degree by the power throughput (10) that a specific design is targeted at, but the process of optimizing the inequality (35) is the same although the layout solution is more complicated than what is illustrated in Fig. 9b. Alternatively the area of the primary electrode can be decreased, without affecting power throughput and conversion ratio.

The ZVS optimization formulated in this paper is based on the ring-shaped PT, but approach it is also valid for rosen type, disc- and retangular-shaped design, where an expression for the conversion ratio (32) and the terminal capacitance (33) can be formulated.

\section{PROTOTYPE PT POWER SUPPLy}

The basis for the practical work done for this paper is based on the $30 \mathrm{~W}$ prototype power supply shown in Fig. 10, which is build around the Noliac 2005-09-05-A PT. As it was verified in Fig. 8 this PT is not designed for native ZVS and this has been confirmed with the experimental work done on the prototype power supply. To avoid excessive losses, a series inductor has been added to the PT. This changes the real and the imaginary part of the input impedance used in the expression for the resonant peak voltage (21) in which ZVS can be achieve. The measured ZVS waveform for the prototype using a series inductor is shown in Fig. 11. 


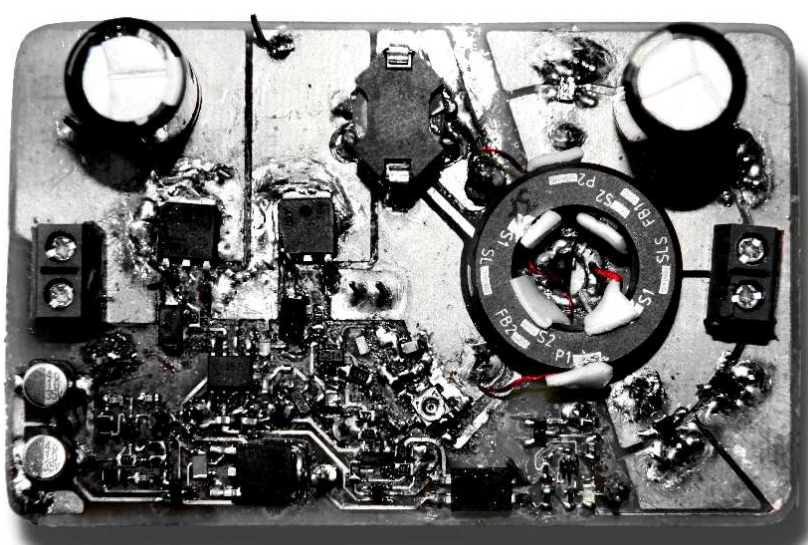

Fig. 10. Prototype Power supply with Noliac 2005-09-05-A PT.

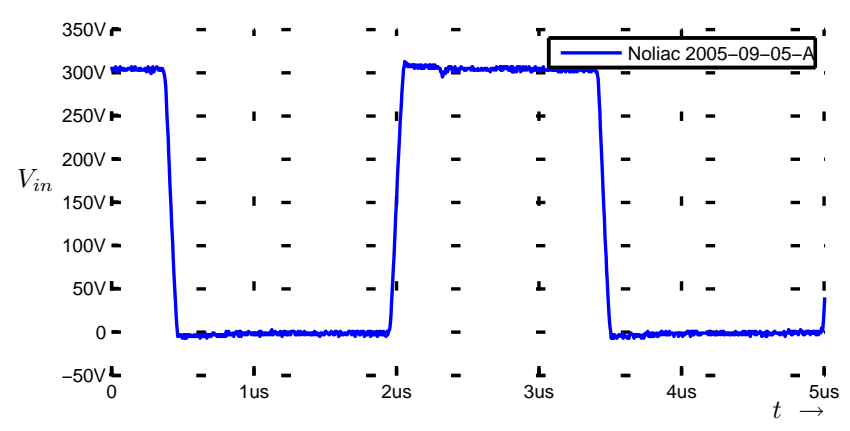

Fig. 11. ZVS waveform with a switching frequency of $329 \mathrm{kHz}$. The series inductor provides very steep soft switching flanks.

\section{CONCLUSION}

A parameterized analysis of ZVS in half bridge resonant power converters has been presented. The approach is par-ticulary useful in conjunction with PTs that can achieve high efficiencies but also suffer from a large inherent parasitic input capacitance that can lead to hard switching losses that are comparable to the power rating of a PT. The derived model define a worst case scenario of the ZVS capabilities of a PT and provide a simple transparent relation between equivalent PT parameters and ZVS properties. As a result it is possible to directly relate desired ZVS properties to the physical electrode layout of a PT, which can be used to aid the design of PTs using CAD software.

The model has been tested with the equivalent parameters of two different PTs and on a complete AC-DC prototype power supply.

\section{REFERENCES}

[1] K. Breboel, "Us patent \#6,707,235: Piezoelectric transformer," Noliac A/S, 2004

[2] J. C. J.Navas, T.Bove and K. Breboel, "Miniaturised battery charger using piezoelectric transformers," Applied Power Electronics Conference and Exposition, 2001. APEC 2001. Sixteenth Annual IEEE, vol. vol.1, pp. 492-496, 2001. Anaheim, CA, USA.

[3] E. R. T Bove, W Wolny and K. Breboel, "New type of piezoelectric transformer with very high power density," Applications of Ferroelectrics, vol. ISAF $2000,2000$.
[4] C. yi Lin, "Design and analysis of piezoelectric transformer converters," ph.d. dissertation, Blacksburg, Virginia, $1997 . \quad$ http://scholar.lib.vt.edu/theses/available/etd-82097163753/unrestricted/diss0917.pdf.

[5] F. L. CY Lin, "Design of a piezoelectric transformer converter and its matchingnetworks," Power Electronics Specialists Conference, PESC'94 Record, 1994.

[6] S. B.-Y. Svetlana Bronstein, "Design considerations for achieving zvs in a half bridge inverter that drives a piezoelectric transformer with no series inductor," Power Electronics Specialists Conference, PESC 02, pp. 585 590 vol. 2, 2002. citeseer.ist.psu.edu/605071.html.

[7] E. M. B. Ray L Lin, Fred C Lee and D. Y. Chen, "Inductor-less piezoelectric transformer electronic ballast for linear fluorescent lamp,' Applied Power Electronics Conference and Exposition, 664-669 vol.2, 2001

[8] R.-L. Lin, "Piezoelectric transformer characterization and application of electronic ballast," ph.d. dissertation, Blacksburg, Virginia, Nov. 26, 2001. http://scholar.lib.vt.edu/theses/available/etd-12062001005832/unrestricted/ETD-RayLeeLin.pdf.

[9] W. Huang, "Design of a radial mode piezoelectric transformer for a charge pump electronic ballast with high power factor and zero voltage switching," master's thesis, Blacksburg, Virginia, April 21, 2003. http://scholar.lib.vt.edu/theses/available/etd-04222003083524/unrestricted/whuangthesisFinal.pdf. 